\title{
Reduction of Seek Time of Tracking Actuator with Pulsed Excitation in Optical Disk
}

\author{
Hyung-Jun LIM, Chang-Soo HAN ${ }^{1}$ and Soo Hyun KIM* \\ Department of Mechanical Engineering, Korea Advanced Institute of Science and Technology, \\ 373-1, Kusong-dong, Yusong-gu, Taejon, 305-701, Republic of Korea \\ ${ }^{1}$ Automation Research Department, Korea Institute of Machinery and Materials, \\ 171, Jang-dong, Yusong-gu, Taejon, 305-343, Republic of Korea
}

(Received April 26, 2000; accepted for publication August 9, 2000)

Time consumption during the track seek is an important factor which indicates the ability of an optical disk system. In a two-stage tracking actuator, latency time exists before the laser beam follows the destination track because of the vibration of the fine actuator. Modified velocity profiles and control techniques for the actuator have been proposed to reduce the latency time. When a fine actuator is locked to the coarse actuator, another latency time caused by the disk eccentricity cannot be reduced. It becomes possible to forecast the eccentricity as the rotation type of the optical disk changes to constant angular velocity (CAV). Thus the latency time can be reduced by excitation of the fine actuator with the same velocity of the track when the coarse actuator stops. Pulsed excitation is used for the excitation. This method is applied to a compact disk read-only memory (CD-ROM).

KEYWORDS: optical disk, tracking actuator, seek time, latency time, disk eccentricity, pulsed excitation

\section{Introduction}

In an optical disk system, the beam spot is usually controlled by a two-stage actuator. The two-stage actuator can be divided into two parts: the fine actuator which can move a short distance of about $1 \mathrm{~mm}$, and the coarse actuator which can move a long distance of about $10-50 \mathrm{~mm}$.

Disk tracks have a width of $0.5 \mu \mathrm{m}$ and a pitch of $1.6 \mu \mathrm{m}$. The oscillation amplitute of the disk track is about $50 \mu \mathrm{m}$, but it increases to $100 \mu \mathrm{m}$ when the disk is placed on a spindle motor.

The rotation type of the spindle motor was changed from constant linear velocity (CLV) to constant angular velocity (CAV). Thus it is possible to forecast the track eccentricity because its frequency is not changed for all track positions.

The fundamental track seek sequence proceeds in the following steps. First, the beam spot is moved to the approximate area of the destination track by the coarse actuator. Due to the vibration of the fine actuator, however, the beam spot cannot immediately follow any track. It is therefore necessary to wait until the velocity difference between the beam spot and a track becomes less than a given value. After this "latency time," the beam spot can follow and read the address of a neighboring destination track. Finally, the beam spot arrives at the destination after the track jump.

Many optimized trajectories and control techniques for the fast coarse movement have been introduced. ${ }^{1,2)}$ Some of these results are also applicable to a hard disk drive (HDD). ${ }^{3,4)}$ There are some papers addressing the minimization of vibration of the fine actuator during the coarse movement. ${ }^{5-7)}$

Even though the vibration is completely eliminated, another latency time due to the track oscillation will remain. It is, therefore, very hard for the beam spot to follow a track after the coarse movement. The latency time can be zero if the velocity of the fine actuator is the same as that of a track. In this study, a method which can eliminate the velocity difference is proposed.

\section{Modeling of a Two-Stage Actuator and Disk Tracks}

Figure 1 shows a schematic diagram of a two-stage actua-

*E-mail address: kimsh@ sorak.kaist.ac.kr tor. $F_{\mathrm{m}}$ and $F_{\mathrm{c}}$ are the forces driving the coarse actuator by a DC motor and the fine one by a voice coil motor (VCM), respectively. $X_{\mathrm{p}}$ is the absolute displacement of the coarse actuator. $X_{\mathrm{f}}$ is the relative velocity of the fine actuator with respect to the coarse one. $X$ is the absolute displacement of the beam spot, and can thus be represented by the sum of $X_{\mathrm{f}}$ and $X_{\mathrm{p}}$. Let $V_{\mathrm{c}}$ be the fine actuator input and $V_{\mathrm{m}}$ the coarse one. Then we can derive the following equations for each actuator:

$$
\begin{aligned}
X_{\mathrm{f}}(s) & =\frac{K_{\mathrm{c}} V_{\mathrm{c}}(s)-M_{\mathrm{f}} s^{2} X_{\mathrm{p}}(s)}{M_{\mathrm{f}} s^{2}+C_{\mathrm{f}} s+K_{\mathrm{f}}} \\
X_{\mathrm{p}}(s) & =\frac{\gamma V_{\mathrm{m}}(s)}{K_{\mathrm{E}} s\left(1+\tau_{\mathrm{M}} s\right)},
\end{aligned}
$$

where $M_{\mathrm{f}}$ is the mass, $C_{\mathrm{f}}$ is the damping coefficient and $K_{\mathrm{f}}$ is the spring constant of the fine actuator. $K_{\mathrm{c}}$ is the constant which is determined by the coil and the magnet of the fine actuator. $K_{\mathrm{E}}$ is the back electromotive force (e.m.f.) constant and $\tau_{\mathrm{M}}$ is the mechanical time constant of the coarse actuator. $\gamma$ is the ratio of $X_{\mathrm{p}}$ to the rotation angle of the motor.

The mass of the fine actuator is negligibly smaller than that of the coarse one. Because the fine actuator is loaded on the coarse one, the acceleration of the coarse one affects that of the fine one; so it easily oscillates when the coarse actuator

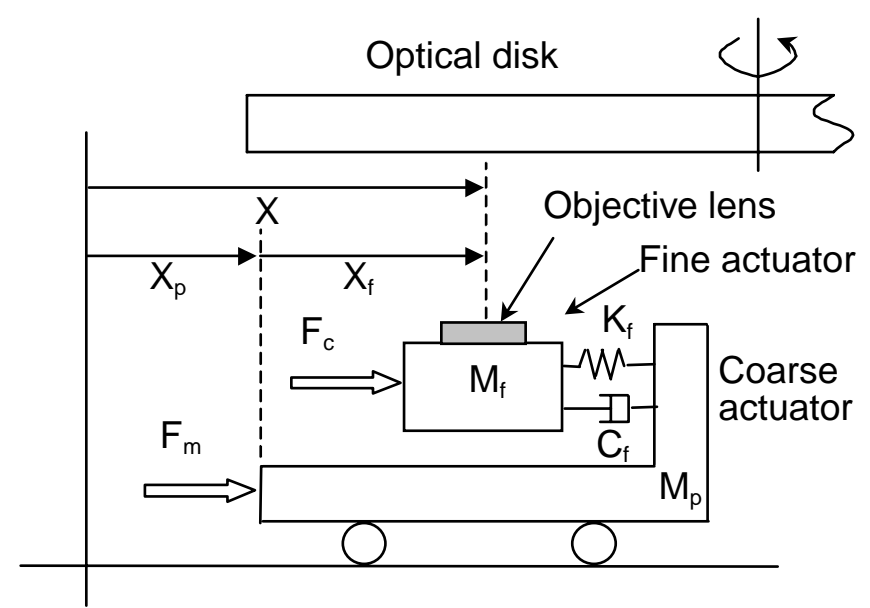

Fig. 1. Schematic diagram of the two-stage actuator. 
moves. This is one of the most severe problems of the track seek.

Let $\omega$ be the angular velocity of the optical disk, and $R_{0}$ and $\phi_{0}$ the amplitude and the phase of the oscillation of tracks, respectively. Then, the velocity of the track oscillation $v_{\mathrm{t}}$ can be represented as

$$
v_{\mathrm{t}}(t)=R_{0} \omega \cos \left(\omega t+\phi_{0}\right),
$$

where $R_{0}$ and $\phi_{0}$ can be sufficiently forecasted by the tracking error signal before the track seek.

\section{Pulsed Excitation with the Feedforward Compensator}

Figure 2 shows a block diagram of the feedforward compensator and the excitation input. $V_{\mathrm{m}}$ is the coarse actuator input. $V_{\mathrm{c}}$ and $V_{\mathrm{E}}$ are the feedforward and the excitation input for the fine actuator, respectively. $V_{\mathrm{c}}$ can be obtained from the coarse actuator model and $V_{\mathrm{m}}$. As well, $V_{\mathrm{E}}$ can be obtained by the fine actuator model and the tracking error signal before the track seek.

The ultimate goal of the feedforward compensator is to have no vibration of the fine actuator from the coarse one by compensating the inertia force. In an ideal case, the latency time can be markedly reduced. Furthermore, it does not need to modify the velocity profile of the coarse actuator. The trapezoidal velocity profile of the coarse actuator is used to minimize coarse moving time.

\subsection{Velocity generation by pulsed excitation}

After unit voltage input is applied to the fine actuator during the time $t_{\mathrm{p}}$, its displacement and velocity become

$$
\begin{aligned}
& x_{\mathrm{jp}}=\frac{K_{\mathrm{c}}}{K_{\mathrm{f}}}\left\{1-e^{-\sigma t_{\mathrm{p}}}\left(\cos \left(\omega_{\mathrm{d}} t_{\mathrm{p}}\right)+\frac{\sigma}{\omega_{\mathrm{d}}} \sin \left(\omega_{\mathrm{d}} t_{\mathrm{p}}\right)\right)\right\} \\
& v_{\mathrm{fp}}=\frac{K_{\mathrm{c}}}{K_{\mathrm{f}}} e^{-\sigma t_{\mathrm{p}}}\left(\frac{\sigma^{2}}{\omega_{\mathrm{d}}}+\omega_{\mathrm{d}}\right) \sin \left(\omega_{\mathrm{d}} t_{\mathrm{p}}\right),
\end{aligned}
$$

where $\omega_{\mathrm{n}}$ is the undamped natural frequency and $\xi$ is the damping ratio of the fine actuator. $\omega_{\mathrm{d}}$ and $\sigma$ are $\omega_{\mathrm{n}} \sqrt{1-\xi^{2}}$ and $\xi \omega_{\mathrm{m}}$, respectively. After the time $t_{\mathrm{r}}$ is passed, the velocity and the acceleration can be represented as follows.

$$
v_{\mathrm{fr}}=e^{-\sigma t_{\mathrm{r}}}\left\{v_{\mathrm{jp}} \cos \left(\omega_{\mathrm{d}} t_{\mathrm{r}}\right)-\frac{\left(\sigma^{2}+\omega_{\mathrm{d}}^{2}\right) x_{\mathrm{fp}}+\sigma v_{\mathrm{fp}}}{\omega_{\mathrm{d}}} \sin \left(\omega_{\mathrm{d}} t_{\mathrm{r}}\right)\right\}
$$

$$
\begin{aligned}
a_{\mathrm{fr}}= & e^{-\sigma t_{\mathrm{r}}}\left[-\left\{\left(\sigma^{2}+\omega_{\mathrm{d}}^{2}\right) x_{\mathrm{fp}}+2 \sigma v_{\mathrm{fp}}\right\} \cos \left(\omega_{\mathrm{d}} t_{\mathrm{r}}\right)\right. \\
& \left.+\frac{1}{\omega_{\mathrm{d}}}\left\{\sigma\left(\sigma^{2}+\omega_{\mathrm{d}}^{2}\right) x_{\mathrm{fp}}+\left(\sigma^{2}-\omega_{\mathrm{d}}^{2}\right) v_{\mathrm{fp}}\right\} \sin \left(\omega_{\mathrm{d}} t_{\mathrm{r}}\right)\right]
\end{aligned}
$$

From eqs. (6) and (7), we can find the minimum positive

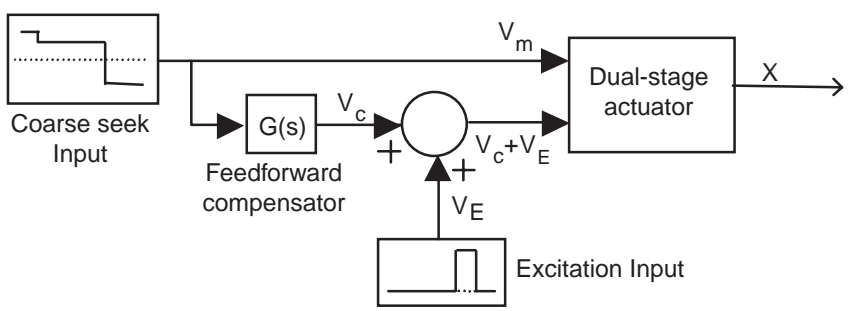

Fig. 2. Block diagram of pulsed excitation with the feedforward compensator.

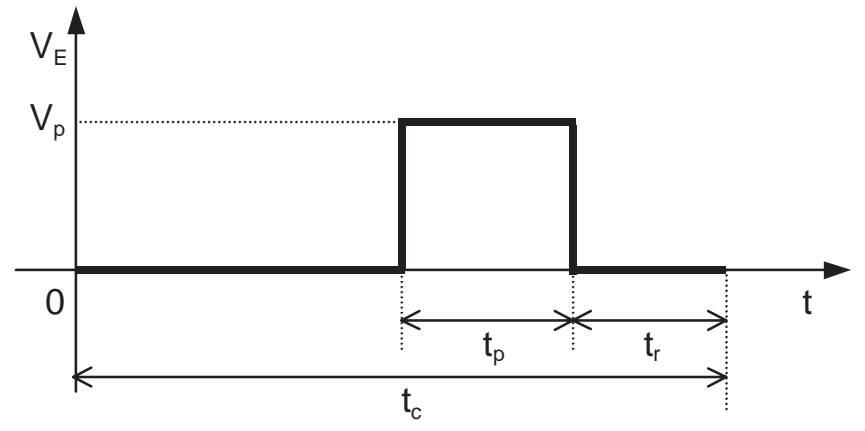

Fig. 3. Excitation input for the two-stage actuator.
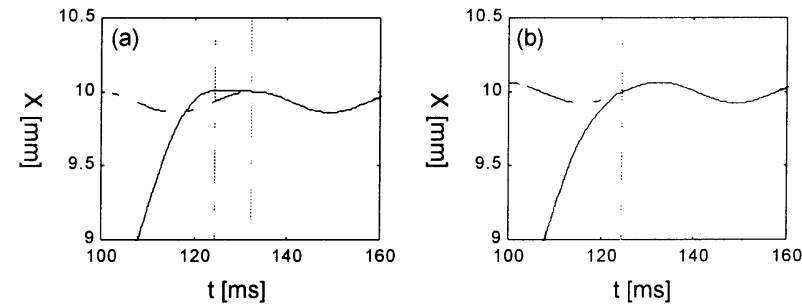

Fig. 4. Beam trajectory (a) without (b) with excitation input.

Table I. Comparison of time consumption.

\begin{tabular}{ccccc}
$\begin{array}{c}\text { Feedforward } \\
\text { compensator }\end{array}$ & $\begin{array}{c}\text { Pulsed } \\
\text { excitation }\end{array}$ & $\begin{array}{c}t_{\mathrm{a}} \\
(\mathrm{ms})\end{array}$ & $\begin{array}{c}t_{\mathrm{l}} \\
(\mathrm{ms})\end{array}$ & $\begin{array}{c}t_{\mathrm{a}}+t_{\mathrm{l}} \\
(\mathrm{ms})\end{array}$ \\
\hline Not used & Not used & 124.3 & 58.5 & 182.8 \\
Used & Not used & 124.3 & 7.6 & 131.9 \\
Used & Used & 124.3 & 0 & 124.3 \\
\hline
\end{tabular}

$t_{\mathrm{r}}$ that makes $a_{\mathrm{fr}}$ zero. This means that there is no velocity change at $t_{\mathrm{r}}$.

The voltage level for the excitation input, $V_{\mathrm{p}}$, which makes $v_{\mathrm{ft}}$ the same as $v_{\mathrm{t}}$ at $t_{\mathrm{c}}$, is shown in eq. (8). $V_{\mathrm{E}}$ in Fig. 2 can be obtained from $t_{\mathrm{p}}, t_{\mathrm{r}}$ and $V_{\mathrm{p}}$, as shown in Fig. 3.

$$
V_{\mathrm{p}}=-\frac{R_{0} \omega \cos \left(\omega t_{\mathrm{c}}+\phi_{0}\right)}{v_{\mathrm{fr}}}
$$

\subsection{Simulation of the track seek}

Figure 4 shows the beam trajectory for two cases: with excitation input or without. The dotted line indicates a disk track and the solid line indicates the beam trajectory. There is no latency time when the excitation input is used with the feedforward compensator. Table I shows the coarse movement time $t_{\mathrm{c}}$, the average latency time $t_{1}$, and the track pull-in time $t_{\mathrm{c}}+t_{1}$ for all cases when the disk rotates at $1800 \mathrm{rpm}$.

The time reduction with the feedforward compensator and the excitation input is $58.5 \mathrm{~ms}$ which is $32 \%$ of the track pull-in time. The effects of the excitation input are $13 \%$.

\section{Experiments and Error Analysis}

Figure 5 shows the experimental setup. The two-stage actuator is controlled by a digital signal processor (DSP). The velocity and displacement profiles can be measured by the optical sensor. 


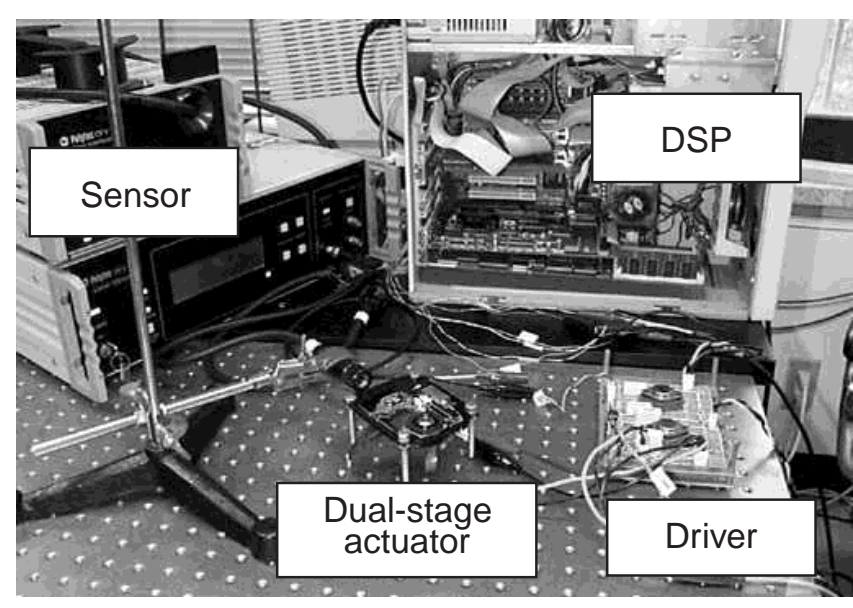

Fig. 5. Experimental setup.

\subsection{Feedforward compensator}

When the feedforward compensator is used, actuator inputs and their velocity profiles are as shown in Fig. 6. There is some difference between the ideal velocity profile of the coarse actuator and the real one, thus the modified feedforward compensation input $v_{\mathrm{c}}^{\prime}$ is used instead of $v_{\mathrm{c}}$. In this experiment, $v_{\mathrm{f}}$ is about $-4-2 \mathrm{~mm} / \mathrm{s}$ during the coarse movement. When the coarse actuator stops, the displacement and the velocity of the fine actuator are $10 \mu \mathrm{m}$ and $-1.22 \mathrm{~mm} / \mathrm{s}$, respectively, and this is sufficiently small. The track pull-in time is $131.9 \mathrm{~ms}$ with the feedforward compensator, and $188.4 \mathrm{~ms}$ without one.

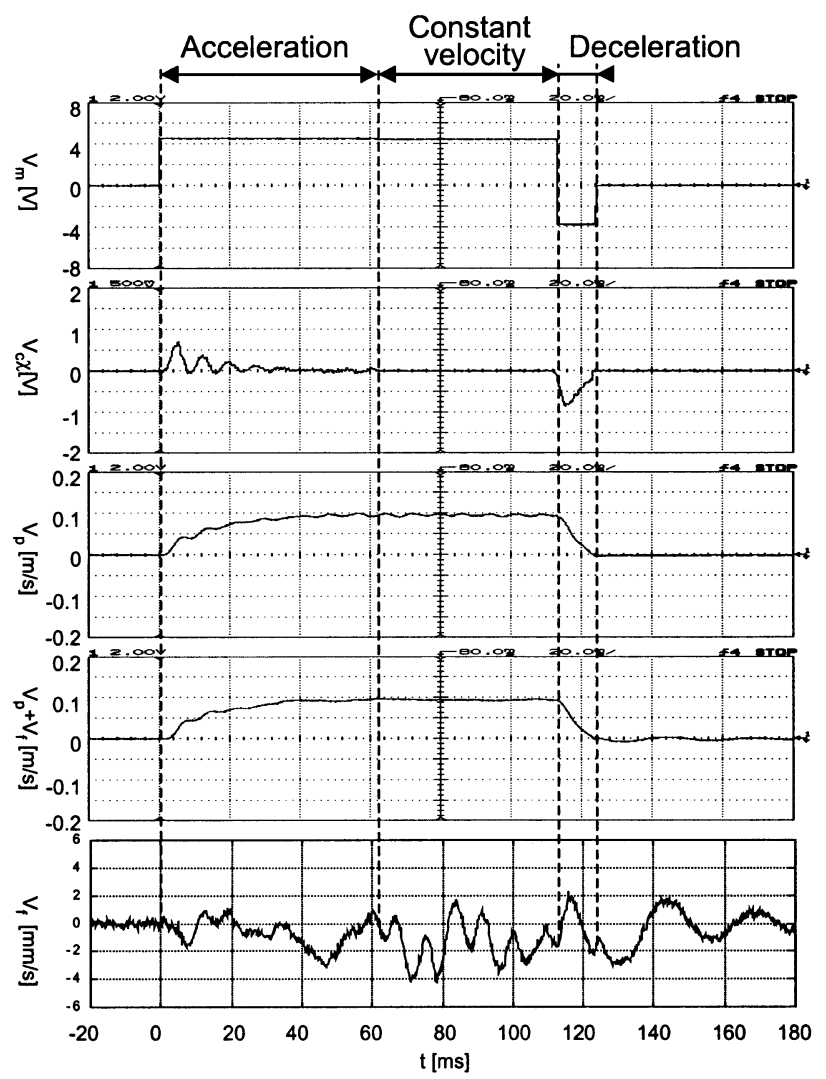

Fig. 6. Experimental result when the feedforward compensator is used.

\subsection{Pulsed excitation with the feedforward compensator}

Figure 7 shows the results of experiments on the excitation of the fine actuator in the case of the maximum track velocity when the excitation input is used. The error of the velocity generation is $0.5 \mathrm{~mm} / \mathrm{s}$. The measured velocity for various desired values is shown in Fig. 8 and Table II. The maximum velocity error is $1.2 \mathrm{~mm} / \mathrm{s}$. The track pull-in time is $124.3 \mathrm{~ms}$ for all cases.

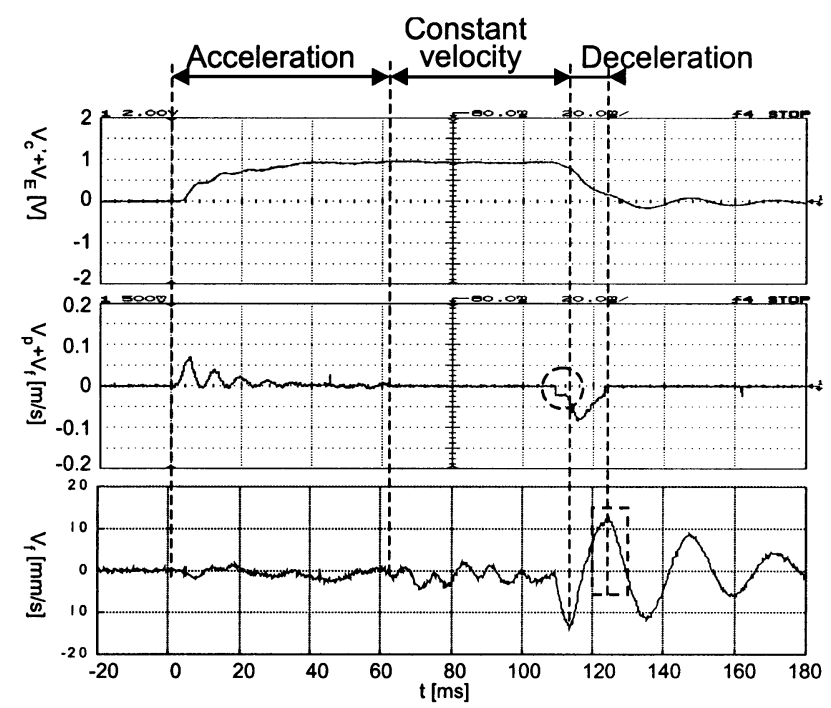

Fig. 7. Experimental result when the feedforward compensator and the excitation input are used.

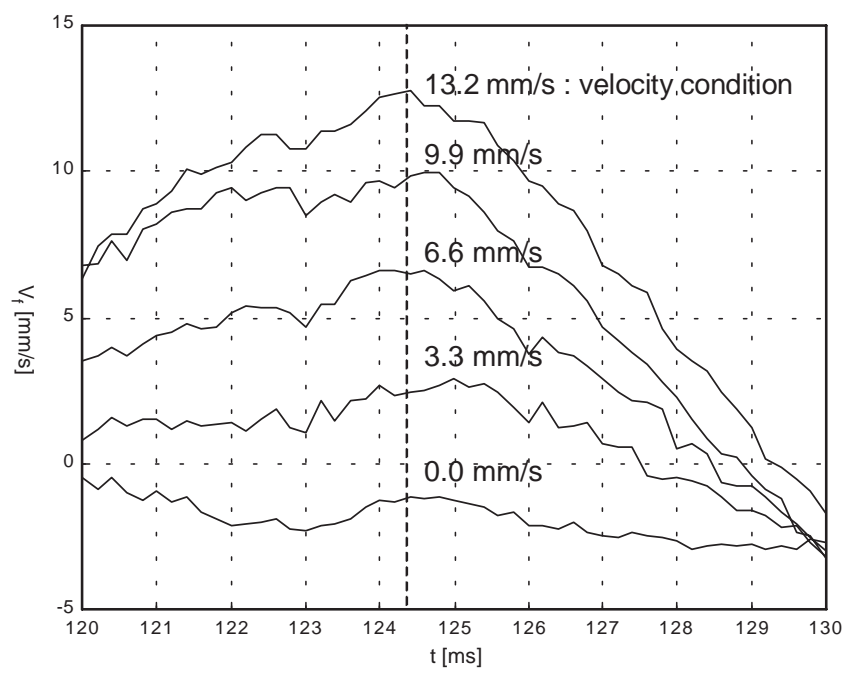

Fig. 8. Velocity variation of the fine actuator according to the velocity condition.

Table II. Velocity for various desired values.

\begin{tabular}{cccccc}
\hline $\begin{array}{c}\text { Desired velocity } \\
(\mathrm{mm} / \mathrm{s})\end{array}$ & 13.2 & 9.9 & 6.6 & 3.3 & 0.0 \\
\hline$x_{\mathrm{f}}(\mu \mathrm{m})$ & 26 & 35 & 31 & 26 & 10 \\
$v_{\mathrm{f}}(\mathrm{mm} / \mathrm{s})$ & 12.7 & 9.7 & 6.6 & 2.4 & -1.2 \\
Velocity error $(\mathrm{mm} / \mathrm{s})$ & -0.5 & -0.2 & 0.0 & -0.9 & -1.2 \\
\hline
\end{tabular}


Table III. Velocity error due to modeling error.

\begin{tabular}{lcc}
\hline \multicolumn{2}{c}{$\begin{array}{c}\text { Maximum } \\
\text { modeling error }\end{array}$} & \multicolumn{1}{c}{$\begin{array}{c}\text { Maximum } \\
\text { velocity error }(\mathrm{mm} / \mathrm{s})\end{array}$} \\
\hline $\begin{array}{c}\text { Resonance } \\
\text { frequency }\end{array}$ & $+2 \mathrm{~Hz}$ & -0.23 \\
\hline \multirow{2}{*}{ Q-factor } & $-2 \mathrm{~Hz}$ & -0.06 \\
\cline { 2 - 3 } & $+3 \mathrm{~dB}$ & +1.26 \\
\cline { 2 - 3 } & $-3 \mathrm{~dB}$ & -1.58 \\
\hline
\end{tabular}

\subsection{Velocity error due to modeling error of the fine actuator}

The fine actuator is a second-order system, as shown in eq. (1). For the fine actuator used in the experiment, $\xi$ and $\omega_{\mathrm{n}}$ are 0.11 and $251 \mathrm{rad} / \mathrm{s}$, respectively.

Some manufacturing errors exist in the natural frequency and the Q-factor. The corresponding velocity errors are shown in Table III. When the Q-factor is $-3 \mathrm{~dB}$, the maximum velocity error is $1.6 \mathrm{~mm} / \mathrm{s}$. This is, however, an allowable value.

\section{Conclusions}

To reduce the track seek time for optical disk systems, many studies on the modification of actuators and controllers have been carried out. It has been assumed that there is no track oscillation. The track eccentricity is mainly considered in this study and it is verified that the corresponding latency time can be reduced by excitation of the fine actuator.

Further studies will investigate the application of this method to the entire optical disk system.

\section{Acknowledgements}

This work was partly supported by the Brain Korea 21 (BK21) Project.

1) J. McCormick and R. Horowitz: Proc. 1990 American Control Conf. (1990) p. 2607.

2) M. Yonezawa, T. Takahara and A. Kasahara: Proc. 3rd Int. Conf. Motion and Vibration Control, 1996, Chiba, p. 154.

3) W. N. Patten, H. C. Wu and L. White: IEEE Trans. Magn. 31 (1995) 2380.

4) J. Ishikawa, M. Hashimoto and T. Tsujisawa: Proc. 2nd Int. Conf. Motion and Vibration Control, 1994, Yokohama, p. 717.

5) K. Kato: Proc. 2nd Int. Conf. Motion and Vibration Control, 1994, Yokohama, p. 728.

6) M. Ogawa, Y. Nakajima, K. Koyanagi and T. Furukawa: Jpn. J. Appl. Phys. 32 (1993) 5376

7) H. Inada, T. Nomura, S. Shimonou and Y. Ichiyama: NEC Res. \& Dev. 71 (1983) 33. 